\title{
Coherent Properties and Rabi Oscillations in Two-Level Donor Systems
}

\author{
A. Latgé ${ }^{1}$, F. J. Ribeiro ${ }^{2}$, A. Bruno-Alfonso ${ }^{3}$, L. E. Oliveira ${ }^{4}$, and H. S. Brandi ${ }^{5}$ \\ ${ }^{1}$ Departamento de Física, Universidade Federal Fluminense,24210-340, Niterói-RJ, Brazil \\ ${ }^{2}$ Depto. de Física, Univ. Estadual de Feira de Santana, 44031-460, Feira de Santana-BA, Brazil \\ ${ }^{3}$ Depto. de Matemática, Fac. de Ciências, Univ. Est. Paulista, 17033-360, Bauru-SP, Brazil \\ ${ }^{4}$ Instituto de Física, Unicamp, CP 6165, 13083-970, Campinas-SP, Brazil and \\ ${ }^{5}$ Instituto de Física, Univ. Federal do Rio de Janeiro, 21945-970, Rio de Janeiro-RJ, Brazil
}

Received on 4 April, 2005

\begin{abstract}
Coherent properties and Rabi oscillations in two-level donor systems, under terahertz excitation, are theoretically investigated. Here we are concerned with donor states in bulk $G a A s$ and $G a A s-(G a, A l) A s$ quantum dots. We study confinement effects, in the presence of an applied magnetic field, on the electronic and on-center donor states in $G a A s-(G a, A l) A s$ dots, as compared to the situation in bulk $G a A s$, and estimate some of the associated decay rate parameters. Using the optical Bloch equations with damping, we study the time evolution of the 1s and $2 p_{+}$states in the presence of an applied magnetic field and of a terahertz laser. We also discuss the role played by the distinct dephasing rates on the photocurrent and calculate the electric dipole transition moment. Results indicate that the Rabi oscillations are more robust as the total dephasing rate diminishes, corresponding to a favorable coherence time.
\end{abstract}

Keywords: Coherent properties; Rabi oscillations; Two-level donor systems

One of the proposals concerning new solid-state quantum computers (QC) is the possibility of using quantum dots (QDs) as the basic architecture for their implementation [1]. In that case, discrete electronic charge or spin states are the qubits responsible for encoding quantum information [2-4]. One crucial point is that the model-qubit system operates under the conditions that decoherence processes are weak and single-qubit and two-qubit unitary operations are controlled. This implies that a QC would be effective only if the decoherence times are much longer than the time involved in the single- and two-qubit operations. The use of laser pulses in controlling the qubit operations may overcome this limitation. Coherent optical excitations in two-level donor systems in bulk GaAs [2], under applied magnetic fields, were converted into deterministic photocurrents. The $1 s$ and $2 p_{+}$ donor states are the model qubits coherently manipulated by laser radiation. A more favorable situation concerning the coherence time may be obtained if the excited donor state lies below the continuum. Donor-doped QDs which exhibit pronounced confining effects are then natural candidates to both theoretical [5] and experimental investigations.

Here we investigate the confinement effects of a model spherical QD, under applied magnetic field, on the electronic and on-center donor states in $G a A s-(G a, A l) A s$ QDs. We investigate the conditions in which one may obtain a bound $2 p_{+}$ state in contrast to the resonant one in the study by Cole et al [2], and using the optical Bloch equations with damping terms [6], we study the time evolution of the $1 \mathrm{~s}$ and $2 p_{+}$donor states under the action of a terahertz laser.

The on-center donor Hamiltonian for a spherical GaAs$(G a, A l) A s \mathrm{QD}$, in the effective-mass approximation, is given by

$$
H=-\nabla^{2}+\gamma l_{z}+\gamma^{2} \rho^{2} / 4+V_{b}(r)-2 / r,
$$

where $l_{z}=\frac{1}{i} \frac{\partial}{\partial \phi}, V_{b}(r)$ is the QD barrier potential, $\gamma=\frac{e \hbar B}{2 m^{*} c R^{*}}=$
$\mu_{B}^{*} B / R^{*}=\left(a_{0}^{*} / l_{B}\right)^{2}$ is the ratio of the magnetic and Coulomb energies (for donors in $G a A s, \gamma=1$ corresponds to an applied magnetic field of $\approx 6.9 T), R^{*} \approx 5.9 \mathrm{meV}$ is the GaAs donor effective Rydberg, $l_{B}=(\hbar c / e B)^{1 / 2}$ is the magnetic length (or cyclotron radius), and $a_{0}^{*}$ and $\mu_{B}^{*}$ are the effective Bohr radius and effective Bohr magneton, respectively. Using hydrogeniclike envelope wave functions [7], the $1 \mathrm{~s}$ and $2 \mathrm{p}_{ \pm}$energies may then be variationally obtained as a function of the $z$-direction applied magnetic field.

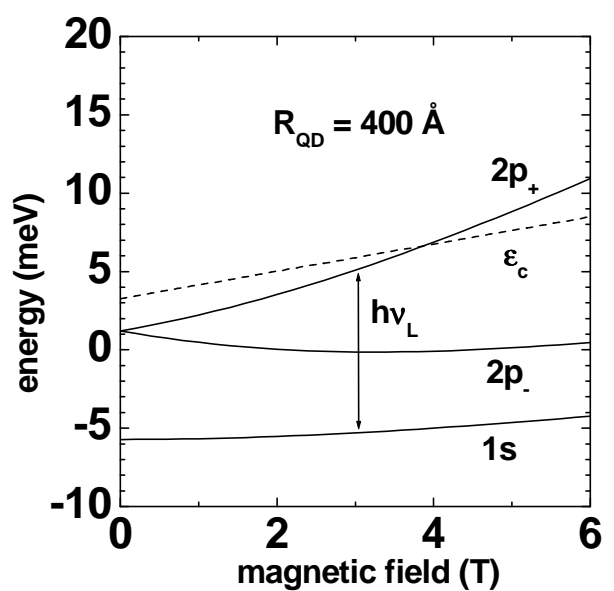

FIG. 1: Magnetic-field dependence of $\varepsilon_{c}=\varepsilon_{o}+\gamma$ and of the energies of $1 \mathrm{~s}, 2 \mathrm{p}_{-}$, and $2 \mathrm{p}_{+}$donor states in a $\mathrm{R}=400 \AA \mathrm{G} a \mathrm{As}-G a_{0.7} A l_{0.3} A s$ QD. The energy $h v_{L}$ for a $2.52 \mathrm{THz}$ free-electron laser is shown as a $1 \mathrm{~s}-2 \mathrm{p}_{+}$transition.

The magnetic-field dependence of the energies of donor states $1 \mathrm{~s}, 2 \mathrm{p}_{-}, 2 \mathrm{p}_{+}$, and of $\varepsilon_{c}=\varepsilon_{0}+\gamma$, for a $R=400 \AA$ $G a A s-G a_{0.7} A l_{0.3} A s$ spherical QD are shown in Fig. 1. Notice that $\varepsilon_{0}$ is the energy of the lowest confined non-occupied electronic state. The arrow shows the $1 \mathrm{~s}-2 \mathrm{p}_{+}$transition energy 

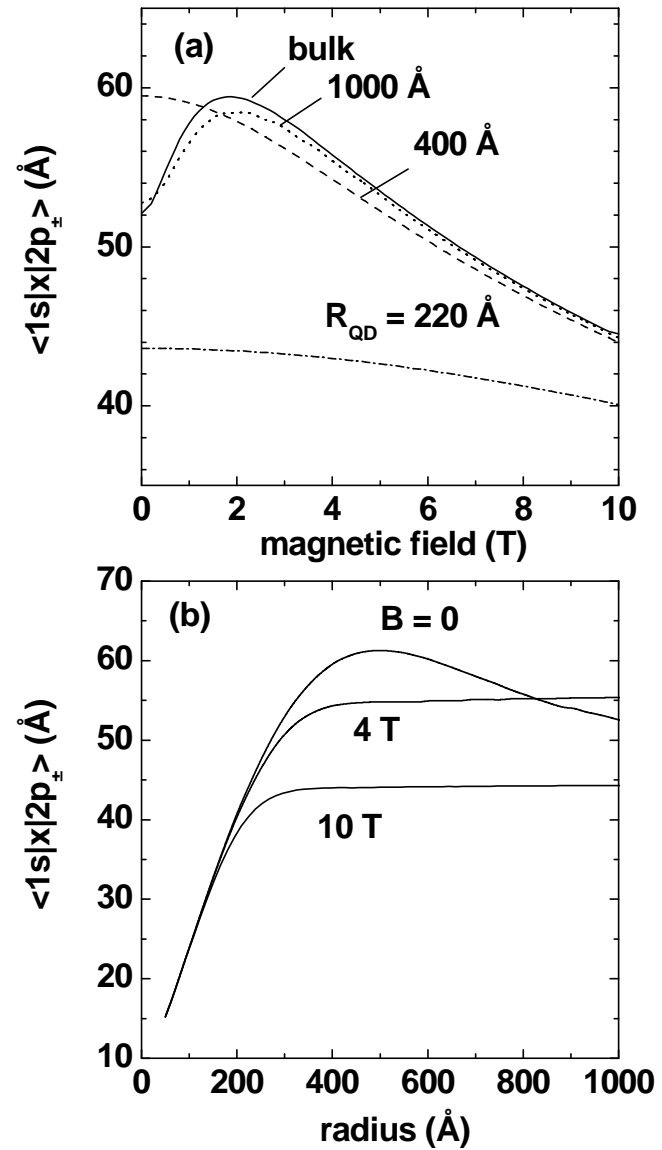

FIG. 2: Magnetic-field (a) and radius (b) dependence of the $\left\langle 1 s|x| 2 p_{ \pm}\right\rangle$matrix elements for bulk GaAs and different GaAs$G a_{0.7} A l_{0.3} A s$ spherical QDs.

corresponding to $2.52 \mathrm{THz}$, which is the free-electron laser frequency used in the experimental measurements by Cole $e t$ al [2]. The confinement effects due to the QD are such that a magnetic field of $\approx 3.0 \mathrm{~T}$ tunes the $\mathrm{THz}$ radiation to the corresponding $1 s-2 p_{+}$transition, with the $2 \mathrm{p}_{+}$below the continuum states, and this source of decoherence is removed [2].

The $x$-component of the corresponding $1 \mathrm{~s}-2 \mathrm{p}_{ \pm}$dipole matrix element, $d_{12}^{x}=\left\langle 1 s|x| 2 p_{ \pm}\right\rangle$, and the Rabi frequency $\Omega_{R}=$ $E_{T H z} d_{12}^{x} / \hbar$, where $E_{T H z}$ is the amplitude of the terahertz electric field (in the $x$-direction), are then calculated. Fig. 2 shows the $\left\langle 1 s|x| 2 p_{ \pm}\right\rangle$matrix elements as a function of the applied magnetic field and of the dot radius. Notice that the $d_{12}^{x}$ matrix-elements results for a $G a A s-G a_{0.7} A l_{0.3} A s$ spherical $\mathrm{QD}$ of radius $\mathrm{R}=1000 \AA$ are essentially the same as for bulk GaAs, as expected. In the bulk regime, for small values of applied magnetic fields, the $\left\langle 1 s|x| 2 p_{ \pm}\right\rangle$matrix elements increase with increasing magnetic fields which can be related to the magnetic-field confinement effects being stronger for the $2 p_{ \pm}$state as compared to the $1 s$ state. This leads to a larger overlap between $1 s-$ and $2 p_{ \pm}$-like wave functions and therefore to a larger value of the $d_{12}^{x}$ matrix-elements. One notices the existence of a maximum around $2-3 T$, which may be

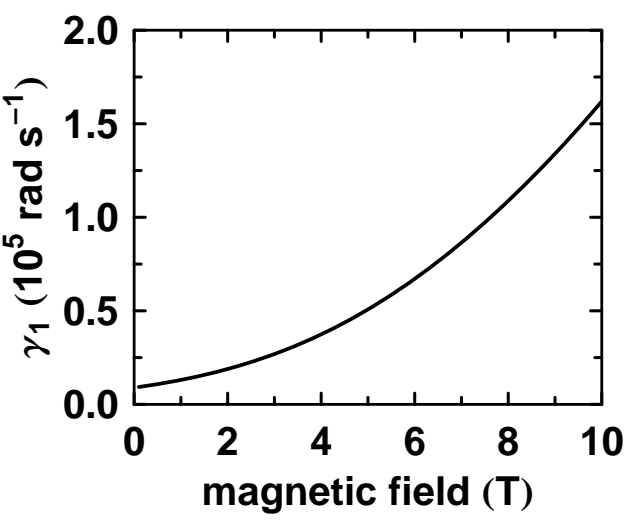

FIG. 3: $1 \mathrm{~s}-2 \mathrm{p}_{+}$recombination rate $\left(\gamma_{1}\right)$ as a function of the magnetic field for a $\mathrm{R}=400 \AA \mathrm{GaAs}-G a_{0.7} A l_{0.3} A s \mathrm{QD}$.

traced back to the fact that, with increasing values of the magnetic field, the Landau magnetic length and Bohr radius (i.e., magnetic and Coulomb energies) become comparable.

The time evolution of the elements of the density matrix within a two-level model for the donor-QD system are obtained via standard procedures $[5,6]$, from the set of optical Bloch equations, i.e.,

$$
\begin{aligned}
& \frac{d \rho_{11}}{d t}=-i \Omega_{R} \cos \left(\omega_{L} t\right)\left(\rho_{21}-\rho_{12}\right)+\gamma_{1} \rho_{22} \\
& \frac{d \rho_{22}}{d t}=+i \Omega_{R} \cos \left(\omega_{L} t\right)\left(\rho_{21}-\rho_{12}\right)-\left(\gamma_{1}+\gamma_{3}\right) \rho_{22} \\
& \frac{d \rho_{12}}{d t}=+i \omega_{21} \rho_{12}+i \Omega_{R} \cos \left(\omega_{L} t\right)\left(\rho_{11}-\rho_{22}\right)-\gamma_{2} \rho_{12} \\
& \frac{d \rho_{21}}{d t}=-i \omega_{21} \rho_{21}-i \Omega_{R} \cos \left(\omega_{L} t\right)\left(\rho_{11}-\rho_{22}\right)-\gamma_{2} \rho_{21}
\end{aligned}
$$

where $\omega_{L}$ is the THz laser frequency, and $\omega_{21}$ is the energy separation of the $1 \mathrm{~s}$ and $2 \mathrm{p}_{+}$impurity levels. The parameters $\gamma_{1}, \gamma_{2}$, and $\gamma_{3}$ are recombination rates as introduced phenomenologically in Cole et al [2].

To calculate the time evolution of the photosignal corresponding to the $1 \mathrm{~s}-2 \mathrm{p}_{+}$transition, we first estimate the recombination rates. The parameter $\gamma_{1}$, giving the rate of spontaneous emission of photons due to $2 p_{+} \rightarrow 1$ s transitions, may be obtained by

$$
\gamma_{1}=\frac{2 e^{2}}{3 \pi \varepsilon_{o} \hbar c^{3}} \omega_{21}^{3}\left|\left\langle 1 s|x| 2 p_{+}\right\rangle\right|^{2} .
$$

Figure 3 shows that the $1 \mathrm{~s}-2 \mathrm{p}_{+}$recombination rate for the $\mathrm{R}=$ $400 \AA \mathrm{GaAs}-G a_{0.7} A l_{0.3} A s$ QD increases with the magnetic field. However, the calculated values are negligible in the $\mathrm{THz}$ range of the oscillation frequencies of the problem, and may be neglected. The dephasing rate $\gamma_{2}$ at the lowest $\mathrm{THz}$ field is estimated from far-infrared measurements as $\gamma_{2}=6.0 \times 10^{10}$ $\operatorname{rad~s}^{-1}$ [8], and the ionization rate $\gamma_{3}$ is set as $\gamma_{3}=0$, since the $2 \mathrm{p}_{+}$excited donor state lies below the first Landau level [2].

Calculated results are shown in Fig. 4 (a), at resonance, for a $\mathrm{R}=400 \AA \mathrm{GaAs}-G a_{0.7} A l_{0.3} A s$ spherical $\mathrm{QD}(\mathrm{B} \approx 3.0 T$ 

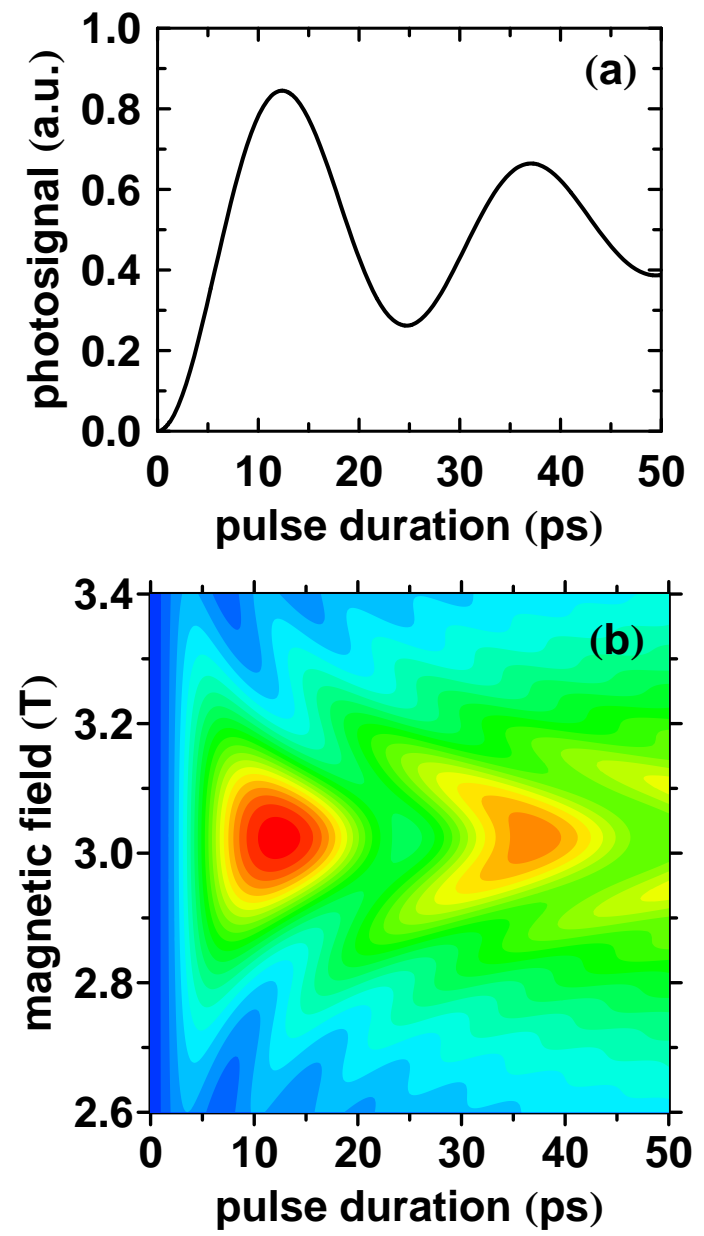

FIG. 4: (a) Theoretical $1 \mathrm{~s}-2 \mathrm{p}_{+}$Rabi oscillations, at resonance, for a fixed THz electric field in the case of a $\mathrm{R}=400 \AA \mathrm{GaAs}-G a_{0.7} A l_{0.3} A s$ spherical QD; (b) Contour plot of photocurrent versus pulse duration and magnetic field at the same fixed THz electric field $\left(\gamma_{1,3}=0\right.$ and $\gamma_{2}=0.6 \times 10^{11} \mathrm{rad} / \mathrm{s}$ ). The THZ field is $E_{T H z}=3 \times 10^{4} \mathrm{~V} / \mathrm{m}$.

and $\left.E_{T H z}=3 \times 10^{4} \mathrm{~V} / \mathrm{m}\right)$. One clearly notices that the displayed Rabi oscillations are more robust, as compared with the experiment by Cole et al [2] in doped bulk GaAs, and therefore it suggests that a donor-doped QD leads to a favorable coherence time so that qubit operations may be efficiently controlled. The corresponding contour plot of the photocurrent for varying pulse duration and applied magnetic field is depicted in Fig. 4 (b).

In summary, we have discussed the possible conditions under which decoherence is weak and qubit operations are efficiently controlled in QDs. Using the optical Bloch equations with damping, we are able to investigate, in a phenomenolog- ical manner, the coherence effects on Rabi oscillations associated to donor states confined in $G a A s-(G a, A l) A s$ QDs in the presence of an applied magnetic field and under a terahertz laser. The pronounced confining effects of semiconductor QDs are shown to provide better coherence-time conditions for the Rabi oscillations.

\section{Acknowledgments}

The authors would like to thank the Brazilian Agencies CNPq, FAPERJ, FUJB, FAPESP, and the Institutes of Millenium (MCT) for partial finantial support.
[1] A. Barenco, D. Deustch, A. Eckert, and R. Jozsa, Phys Rev. Lett. 74, 4083 (1995); G. D. Sanders, K. W. Kim,W. C. Holton, Phys. Rev. A 60, 4146 (1999); M. S. Sherwin, A. Imamoglu, and T. Montroy, Phys. Rev. A 60, 3508 (1999); X.-Q. Li and Y. Arakawa, Phys. Rev. A 63, 012302 (2000); X. Hu and S. Das Sarma, Phys. Rev. A 61, 062301 (2000); X.-Q. Li and Y. Yan, 
Phys. Rev. B 65, 205301 (2002).

[2] B. E. Cole, J. B. Williams, B. T. King, M. S. Sherwin, and C. R. Stanley, Nature 410, 60 (2001).

[3] A. Zrenner, E. Beham, S. Stufler, F. Findels, M. Bichler and G. Abstreiter, Nature 418, 612 (2002); E. Beham, A. Zrenner, F. Findels, M. Bichler and G. Abstreiter, Phys. Stat. Sol. (b) 238, 366 (2003).

[4] X. Li, Y. Wu, D. G. Steel, D. Gammon, T. H. Stievater, D. S. Katzer, D. Park, C. Piermarocchi and L. J. Sham, Science 301, 809 (2003).
[5] H. S. Brandi, A. Latgé, and L. E. Oliveira, Phys. Rev. B 64, 233315 (2001); ibid. 68, 233206 (2003).

[6] C. Cohen-Tannoudju, J. Dupont-Roc, and G. Grynberg, Processus d'Interaction entre Photons et Atomes (Editions du CNRS, Paris, 1988).

[7] L. H. M. Barbosa, A. Latgé, M. de Dios-Leyva, and L. E. Oliveira, Sol. State Commun. 98, 215 (1996).

[8] H. Kobori, M. Inoue, and T. Ohyama, Physica B 302, 17 (2001). 